\title{
Face segregation and recognition by cortical multi-scale line and edge coding
}

\author{
J. Rodrigues ${ }^{1}$ and J.M.H. du Buf ${ }^{2}$ \\ ${ }^{1}$ University of Algarve, Escola Superior Tecnologia, 8005-139 Faro, Portugal \\ ${ }^{2}$ University of Algarve, Vision Laboratory - FCT, 8005-139 Faro, Portugal \\ \{jrodrig, dubuf\}@ualg.pt
}

\begin{abstract}
Models of visual perception are based on image representations in cortical area V1 and higher areas which contain many cell layers for feature extraction. Basic simple, complex and end-stopped cells provide input for line, edge and keypoint detection. In this paper we present an improved method for multi-scale line/edge detection based on simple and complex cells. We illustrate the line/edge representation for object reconstruction, and we present models for multi-scale face (object) segregation and recognition that can be embedded into feedforward dorsal and ventral data streams (the "what" and "where" subsystems) with feedback streams from higher areas for obtaining translation, rotation and scale invariance.
\end{abstract}

\section{Introduction}

Our visual system is by far the most complicated but also least understood part of our body. At the retinal level, already twelve types of different bipolar cells have been identified. These perform complex inhibitory and excitatory interactions in the inner plexiform layer [1]. Apart from the rods and cones, the common photoreceptors, retinal ganglion cells have been identified that have no (in)direct connection to rods and cones, but their dendrites act as photoreceptors. These ganglion cells transfer luminance information to central brain regions, including the LGN (lateral geniculate nucleus), for controlling the circadian clock (solar day), the eyes' iris muscles (pupil size), and probably for providing a global background in brightness perception [2]. The LGN was long considered to be a simple relay station between retinal ganglion cells and the primary visual cortex. Recent research shows that perhaps only 10\% of LGN input comes from the retinas, i.e. the other $90 \%$ consist of feedback from cortical areas, and that the LGN may play a role as information "gatekeeper" through visual attention [3]. The latter finding implies that the what and where subsystems, which are thought to be driven by short-term memory in inferior-temporal (IT) and prefrontal (PF) areas, have feedback loops to areas V4, V2 and V1 [4,5], even down to the LGN.

In V1 there are simple and complex cells, which are tuned to different spatial frequencies (scales) and orientations, but also disparity (depth) because of the left-right hypercolumns, e.g. [6,7]. These cells provide input for grouping cells that code line and edge information and that attribute depth information. There also are end-stopped cells that, together with complicated inhibition processes, allow to extract keypoints 
(singularities, vertices and points of high curvature). Recently, models of simple, complex and end-stopped cells have been developed, e.g. [8]. In addition, several inhibition models, e.g. [9], keypoint detection [8, 10] and line/edge detection schemes [9-12], including disparity models $[13,10]$, have become available. On the basis of these models and neural processing schemes, it is now possible to create a cortical architecture for figure-ground segregation, e.g. [14], and Focus-of-Attention (FoA), bottom-up and/or top-down $[4,15]$, including object detection and recognition.

A difficult and still challenging application, even in machine vision, is face detection and recognition. Despite the impressive number and variety of methods devised for faces and facial landmarks, e.g. [16], complicating factors that still remain are pose (frontal vs. profile), beards, moustaches and glasses, facial expression and image conditions (lighting, resolution). Despite these complications, it is important to develop cortical models for detection and recognition, in order to improve our insight into possible, neural processing schemes, e.g. [17].

In this paper we will focus exclusively on the multi-scale line/edge representation based on simple and complex cells in V1, a representation which is common in perception models but not in computer vision. We present an improved model for line and edge detection, and we apply this within the context of a cortical architecture, the where and what systems, with an emphasis on face (object) detection and recognition.

We stress that we will not employ the multi-scale keypoint representation that also exists in area V1, in order to study the importance of the line/edge representation. It has been shown, e.g. [15], that keypoints alone provide very important information for FoA, object categorisation and facial landmark detection. Also, we will not tackle complications referred to above, because we will argue, in the Discussion, that low-level processing in area V1 needs to embedded into a much wider context, and this context is expected to solve many problems.

\section{Line/Edge Detection and Classification}

Gabor quadrature filters provide a model of receptive fields (RFs) of cortical simple cells (see e.g. $[9,15]$ ). We apply filters with an aspect ratio of 0.5 and half-response width of one octave. The scale $s$ will be given by $\lambda$, the wavelength, in pixels (all images shown are of size $256 \times 256$ pixels). Responses of even and odd simple cells, which correspond to the real and imaginary parts of a Gabor filter, are denoted by $R_{s, i}^{E}(x, y)$ and $R_{s, i}^{O}(x, y), s$ being the scale, $i$ the orientation $\left(\theta_{i}=i \pi /\left(N_{\theta}-1\right)\right)$ and $N_{\theta}$ the number of orientations (here we use $N_{\theta}=8$ ). Responses of complex cells are modelled by the modulus $C_{s, i}(x, y)=\left[\left\{R_{s, i}^{E}(x, y)\right\}^{2}+\left\{R_{s, i}^{O}(x, y)\right\}^{2}\right]^{1 / 2}$.

A basic scheme for single-scale line and edge detection based on responses of simple cells works as follows [12]: a positive (negative) line is detected where $R^{E}$ shows a local maximum (minimum) and $R^{O}$ shows a zero crossing. In the case of edges the even and odd responses are swapped. This gives 4 possibilities for positive and negative events. An improved scheme [10] consists of combining responses of simple and complex cells, i.e. simple cells serve to detect positions and event types, whereas complex cells are used to increase the confidence. Since the use of Gabor modulus (complex 
cells) implies a loss of precision at vertices [18], increased precision was obtained by considering multiple scales (neighbouring micro-scales).

The algorithms described above work reasonably well but there remain a few problems: (a) either one scale is used or only a very few scales for increasing confidence, (b) some parameters must be optimised for specific input images or even as a function of scale, (c) detection precision can be improved, and (d) detection continuity at curved lines/edges must be guaranteed.

We present an improved algorithm with no free parameters, truly multi-scale, with new solutions for problems (c) and (d). With respect to precision, simple and complex cells respond beyond line and edge terminations, for example beyond the corners of a rectangle. In addition, at line or edge crossings, detection leads to continuity of the dominant events and gaps in the sub-dominant events. These gaps must be reduced in order to reconstruct continuity. Both problems can be solved by introducing new inhibition schemes, like the radial and tangential ones used in the case of keypoint operators [10]. Here we use lateral (L) and cross-orientation (C) inhibition, defined as:

$$
\begin{array}{r}
I_{s, i}^{L}(x, y)=\left[C_{s, i}\left(x+d \mathcal{C}_{s, i}, y+d \mathcal{S}_{s, i}\right)-C_{s, i}\left(x-d \mathcal{C}_{s, i}, y-d \mathcal{S}_{s, i}\right)\right]^{+}+ \\
{\left[C_{s, i}\left(x-d \mathcal{C}_{s, i}, y-d \mathcal{S}_{s, i}\right)-C_{s, i}\left(x+d \mathcal{C}_{s, i}, y+d \mathcal{S}_{s, i}\right)\right]^{+}} \\
I_{s, i}^{C}(x, y)=\left[C_{s,\left(i+N_{\theta} / 2\right)}\left(x+2 d \mathcal{C}_{s, i}, y+2 d \mathcal{S}_{s, i}\right)-2 . C_{s, i}(x, y)+\right. \\
\left.C_{s,\left(i+N_{\theta} / 2\right)}\left(x-2 d \mathcal{C}_{s, i}, y-2 d \mathcal{S}_{s, i}\right)\right]^{+}
\end{array}
$$

where $\left(i+N_{\theta} / 2\right) \perp i$, with $\mathcal{C}_{s, i}=\cos \theta_{i}$ and $\mathcal{S}_{s, i}=\sin \theta_{i}$ and $d=0.6 s$. Inhibition is applied to complex cell responses, where $\beta$ controls the strength of the inhibition (we use $\beta=1.0)$, i.e. $\hat{C}_{s, i}(x, y)=\left[C_{s, i}(x, y)-\beta\left\{I_{s, i}^{L}(x, y)+I_{s, i}^{C}(x, y)\right\}\right]$.

Line/edge detection is achieved by constructing a few cell layers on top of simple and complex cells. The first layer serves to select active regions and dominant orientations. At each position, responses of complex cells are summed $\left(\hat{C}_{s}=\sum_{i=0}^{N_{\theta}-1} \hat{C}_{s, i}\right)$, and at positions where $\hat{C}_{s}>0$ an output cell is activated. At active output cells, the dominant orientation is selected by gating one complex cell on the basis of nonmaximum suppression of $\hat{C}_{s, i}$. The gating is confirmed or corrected by an excitation/inhibition process of dominant orientations in a local neighborhood.

In the second layer, event type and position are determined on the basis of active output cells (1st layer) and gated simple and complex cells. A first cell complex checks simple cells $R_{s, i}^{E}$ and $R_{s, i}^{O}$ for a local maximum (or minimum by rectification) using a dendritic field size of $\pm \lambda / 4, \lambda$ being the wavelength of the simple cells (Gabor filter). The active output cell is inhibited if there is no maximum or minimum. A second cell complex does exactly the same on the basis of complex cells. A third cell complex gates four types of zero-crossing cells on the basis of simple cells, again on $\pm \lambda / 4$. If there is no zero-crossing, the output cell is inhibited. If there is a zero-crossing, the active output cell at the position of the zero-crossing cell determines event position and the active zero-crossing cell determines event type.

In the third layer, the small loss of accuracy due to the use of complex cells in the second layer is compensated. This is done by correcting local event continuity, considering the information available in the second layer, but by using excitation of output cells 

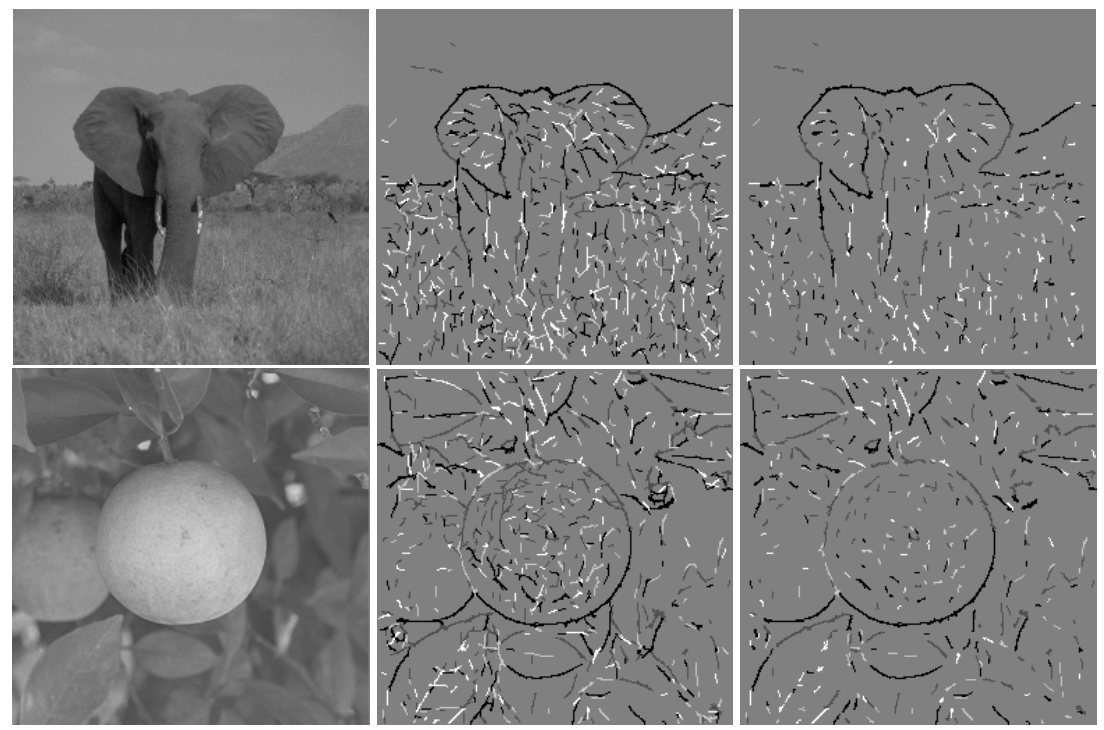

Fig. 1. Fine-scale line/edge detection and NCRF inhibition (right).

by means of grouping cells that combine simple and complex cells tuned to the same and two neighbouring orientations. The latter process is an extension of linear grouping [12] and a simplification of using banana wavelets [19]. In the same layer, event type is corrected in small neighborhoods, restoring type continuity, because the cell responses may be distorted by interference effects when two events are very close [18].

Figure 1 shows detection results with positive and negative lines and edges coded by different gray levels. Detection accuracy is very good and there remain many small events due to low-contrast textures and the fact that no threshold value has been applied (no event amplitudes are shown!). Most events in textured regions can be suppressed by NCRF inhibition (last column). For comparing results (elephant image) obtained with NCRF we refer to [9], but we note that they developed contour (edge) detection algorithms, whereas we can distinguish between edges and lines with different polarities, which is necessary for visual reconstruction; see below. To compare results in the case of the orange image with edge-only computer vision algorithms, i.e. Bergholm, Canny, Iverson and Nalwa, see [20] and http://marathon.csee.usfledge/edge_detection.html.

\section{Multi-scale Representation and Reconstruction}

For illustrating scale space we can create an almost continuous, linear scaling with 288 scales on $\lambda=[4,40]$, but here we will present only a few scales. Figure 2 shows events detected at five scales: $\lambda=\{4,12,18,24,40\}$. In the case of the solid square and star objects, at fine scales (left) the edges of the square are detected, as are most parts of the star, but not at the very tips of the star. This illustrates an important difference between normal image processing and developing cortical models. The latter must be able to 


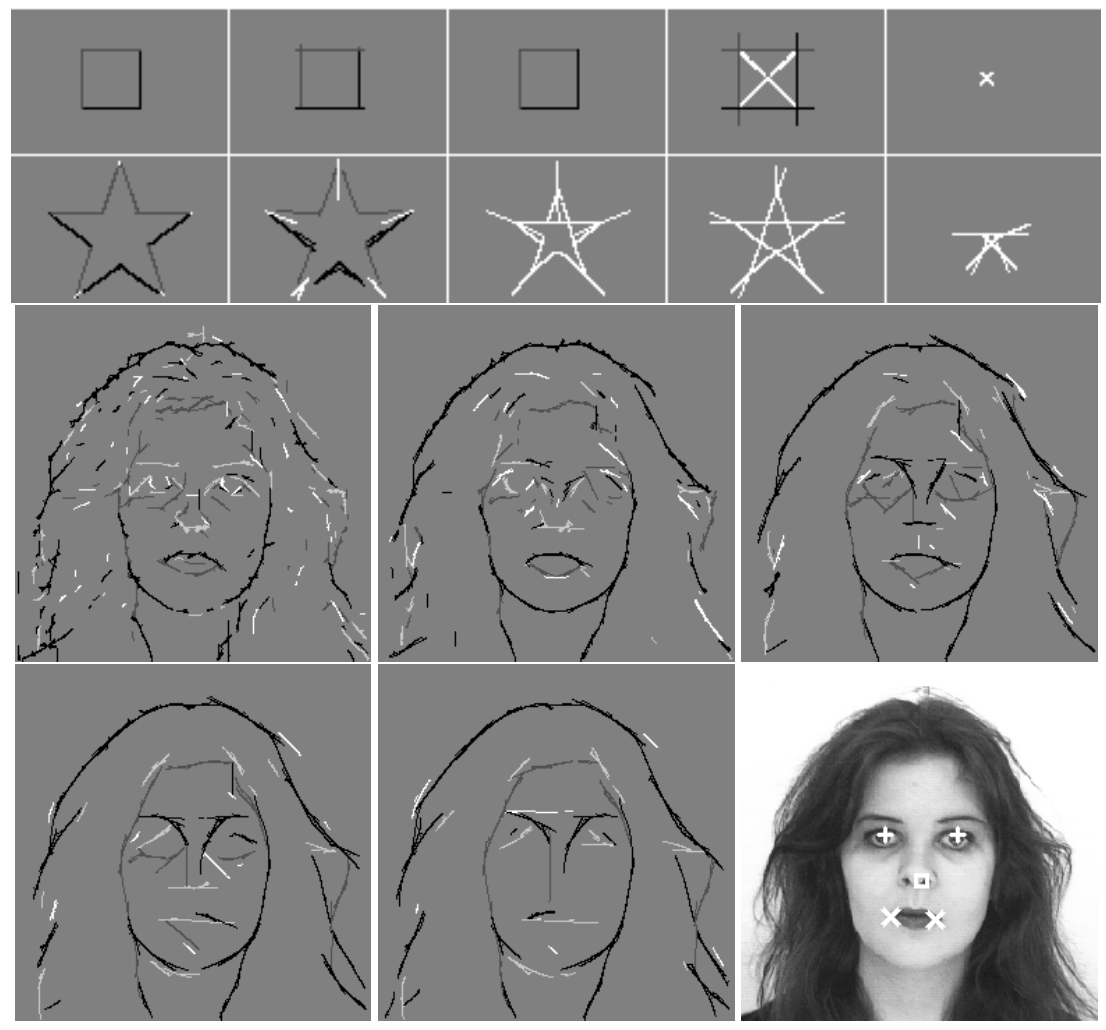

Fig. 2. Multi-scale representations of a square, a star and Fiona image, the latter with detected facial landmarks (see text).

construct brightness maps, and at the tips of the star, where two edges converge, there are very fine lines. The same effect occurs at coarser scales (right), until entire triangles are detected as lines and even pairs of opposite triangles. In the case of the square, lines will be detected at diagonals, which will vanish, with very small event amplitudes, at very coarse scales. Figure 2 (bottom) shows the Fiona image with events detected at the same scales, with decreasing amount of detail at coarser scales but stable outlines of the face and hair-a caricature-like abstraction that is also obtained by other scale spaces, e.g. [21]. Also shown (bottom-right) are facial landmarks used by grouping operators in keypoint scale space for face detection (see Discussion and [15]).

Image reconstruction can be obtained by assuming one lowpass filter plus a complete set of (Gabor) wavelets that cover the entire frequency domain-this concept is exploited in image coding. The goal of our visual system is to detect objects, with no need, nor capacity, to reconstruct a complete image of our visual environment, see change blindness and the limited "bandwidth" of the what and where subsystems [5]. Yet, the image that we perceive in terms of brightness must somehow be created. An image coding scheme, for example by summing responses of simple cells, requires accumulation in one cell layer which contains a brightness map, but this would require 


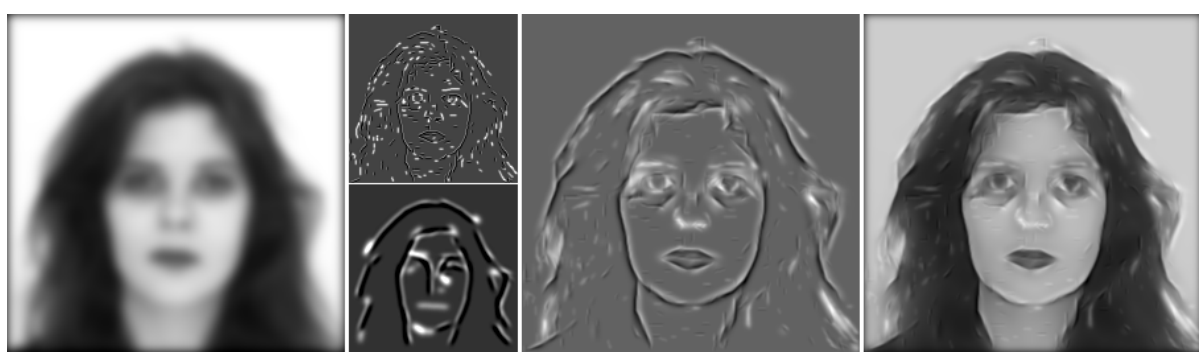

Fig. 3. Reconstruction (see text).

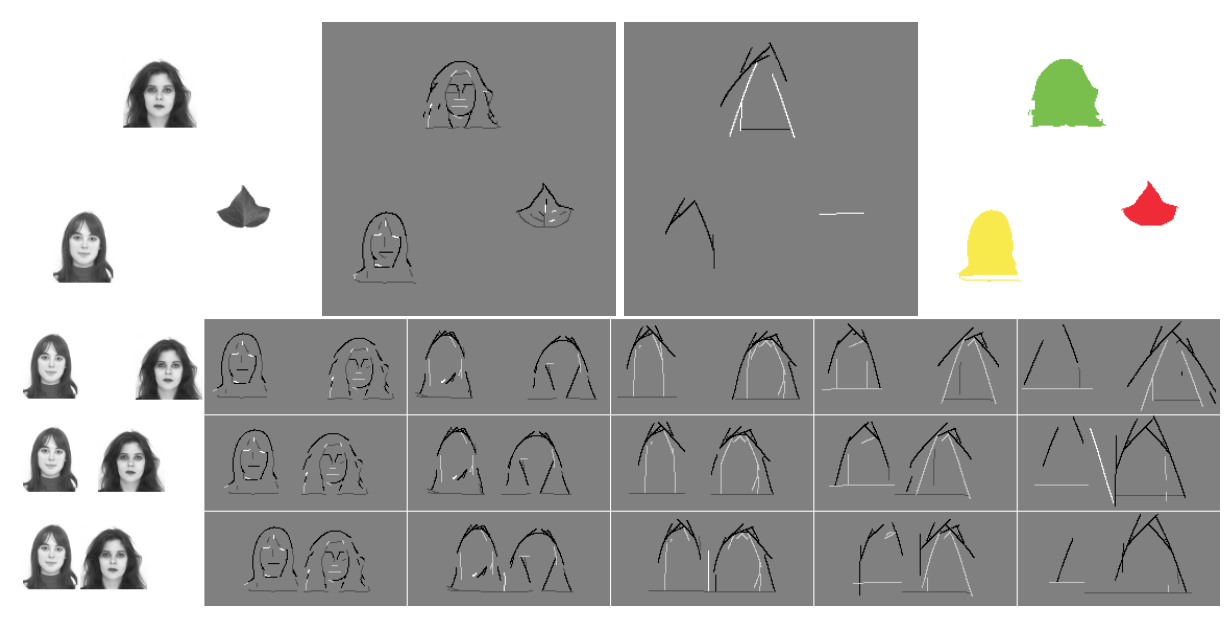

Fig. 4. Top, from left to right: input, representations at $\lambda=4$ and 40, and figure-ground segregation. Bottom: object interference at coarse scales $(\lambda=\{5,15,25,35,45\})$.

yet another "observer" of this map in our brain. The solution to this dilemma is to assume that detected lines and edges are interpreted symbolically: an active "line cell" is interpreted as a Gaussian intensity profile with a certain orientation, amplitude and scale, the size of the profile being coupled to the scale of the underlying simple and complex cells. In the same way an active "edge cell" is interpreted, but with a bipolar, Gaussian-truncated error-function profile. As for image coding, this representation must be complemented with a lowpass filter: the retinal ganglion cells with photoreceptive dendritic fields not (in)directly connected to rods and cones [2].

One brightness model [22] is based on the symbolic line/edge interpretation. It explains Mach bands by the fact that simple cell responses cannot distinguish between lines and ramp edges, and it was shown to be able to predict many brightness illusions such as simultaneous brightness contrast and assimilation, which are two opposite induction effects [23]. Here we will not go into more detail; we will only illustrate the symbolic reconstruction process in 2D (the model referred to above was tested in 1D). Figure 3 shows, from left to right, a lowpass filtered image of Fiona, symbolic line/edge representations at two scales, combined representations over 4 scales, and the latter 
combined with the lowpass image. Using more scales leads to better reconstructions, but the relative weighting of all components requires further investigation.

\section{Object Detection and Segregation}

Until here we have illustrated the multi-scale line/edge detection in area V1 and the symbolic interpretation for reconstruction, but the goal of the visual cortex is to detect and recognise objects by means of the what and where systems. Rensink [5] argued that these systems can attend only one object at any time. In the Deco and Rolls model [4], the ventral what system receives input from V1 which proceeds through V2 and V4 to IT. The dorsal where system connects V1 and V2 through MT to area PP. Both systems are "controlled," top-down, by attention and short-term memory with object representations in PF, i.e. a what component from PF46v to IT and a where component from PF46d to PP. The bottom-up (visual input code) and top-down (expected object and position) data streams are necessary for obtaining size, rotation and position invariance, which means that object templates in memory may be normalised. Here we will not go into more detail, because our goal is not to (re)implement the entire model. Our goal is to show how the line/edge code can be used in the what and where systems, focusing on multi-scale processing.

Figure 4 (top row) shows an image with two faces and a leaf, plus detected events at a fine scale (middle-left), with all relevant information, and at a coarse scale (middleright), with reduced and "deformed" information. At the coarse level, each individual event (group of responding line/edge cells) or connected group of events corresponds to one object. Each event at the coarse scale is related to events at one finer scale, which can be slightly displaced or rotated. This relation is modeled by downprojection using grouping cells with a dendritic field, the size of which defines the region of influence. A responding event cell activates a grouping cell; only if the grouping cell is also excitated by responding event cells one level lower, a grouping cell at the lower level is activated. This is repeated until the finest scale. This coarse-to-fine-scale process is complemented by inhibition: other grouping cells at the finest scale are activated by responding event cells at that scale, and these grouping cells excitate the grouping cells at the one coarser scale. This results in a figure-ground map at the first coarser scale "above" the finest scale (Fig. 4 top-right). This result was obtained with $\lambda=[4,40], \Delta \lambda=4$.

A process as described above can be part of the where system, but it cannot obtain size, rotation and position invariance; it needs to be embedded into the Deco and Rolls model [4]. In addition, when two objects are very close, they will become connected at coarse scales (Fig. 4 bottom row), and separation is only possible by the what system that checks features of individual objects.

\section{Face Recognition}

We assume that templates (views) of faces are stored in memory ${ }^{3}$ and that these have been built through experience. When the where system detects a face, the attentional

\footnotetext{
${ }^{3}$ We will not go into the view-based vs. mental-rotation discussion.
} 
system will direct the what system in order to check features for identification. Here we present a very simple, straightforward recognition scheme for testing the line/edge representation, assuming that input and stored faces are more or less normalised in terms of position and size. From the Psychological Image Collection at Stirling University (UK), we selected 88 face images of 25 persons in frontal and (near) 3/4 view, with different expressions, half against a dark and half against a white background. For typical examples see Fig. 5.

Because small rotations and changes in expression do not significantly change the line/edge representations at coarser scales, and because in practical applications frontal and/or 3/4 views with different expressions may be represented in a database, we created only 25 templates, one for each person, by randomly selecting one of the available images. The multi-scale line/edge representation was computed at 8 scales equally spaced on $\lambda=[4,30]$. Classification was done using all 88 images. For each template, at each scale, a positional relaxation area [24] was created around each responding event cell, by assuming grouping cells with a dentritic field size coupled to the size of underlying complex cells. These grouping cells sum the occurence of events in the input images around event positions in the templates, a sort of local correlation, and activities of all grouping cells were grouped together (global correlation). The final grouping was compared over the 25 templates, scale by scale, and the template with maximum response was selected. Finally, the template with the maximum number of correspondences over the 8 scales was selected (in the case of 4-4 the second template). Figure 5 shows in the upper-left corner of each image 8 bars (scales) with the length of the bar being the number of the selected template (1-25). Despite the extreme simplicity of the method, average recognition rate was $90 \%$ when only grouping events (no discrimination between lines and edges) and $92 \%$ when lines and edges were grouped separately. Misclassifications were only due to extreme expression (Fig. 5 bottom-right) or wrong view

(last images on first and second row), although some images were correctly identified (2nd image on 2nd row and 1st image on 4th row). Also, added noise (4th image on 1 st row) and glasses (4th image on 3rd row) were no problem. Our result (92\%) is very close to the $94 \%$ obtained by Petkov, Kruizinga and Lourens [17].

\section{Discussion}

In this paper we presented an improved scheme for line/edge (event) detection on the basis of simple and complex cells. This scheme has no thresholds nor free parameters, it improves completeness and continuity, and it can be applied at many scales. If necessary, events due to textures can be suppressed by NCRF inhibition [9], such that significant image structures are preserved. We showed that the multi-scale representation and symbolic interpretation are suitable for visual reconstruction, which is necessary for developing a 2D brightness model on the basis of one that was tested in 1D [23]. We also presented simple schemes for object segregation and face recognition.

The line/edge representation at coarser scales leads to a stable abstraction of image features (Fig. 2). This explains, at least partly, the generalisation that allows to classify faces with noise, glasses, and relatively normal expressions and views (Fig. 5). It should be stressed that the recognition scheme is not yet complete, because a hierar- 

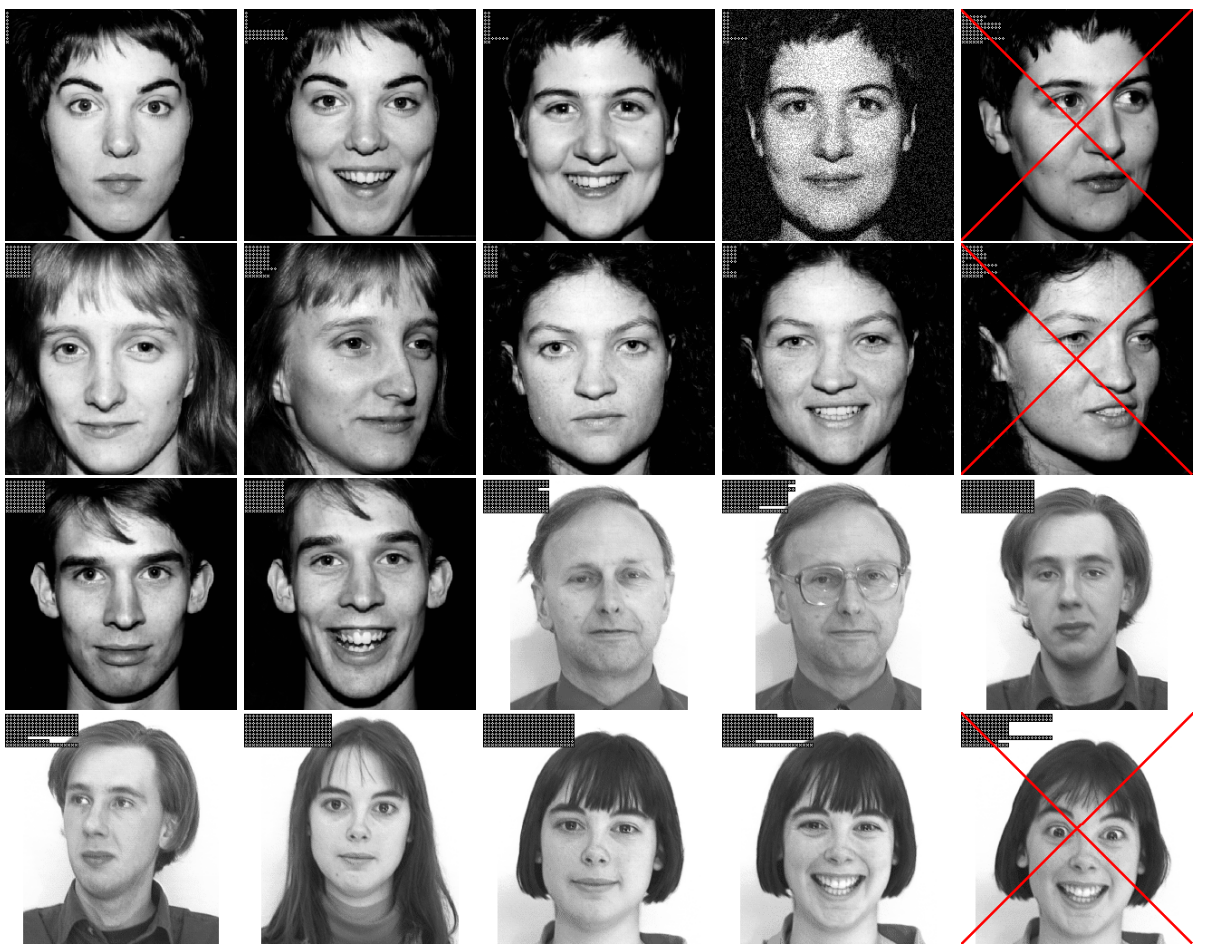

Fig. 5. Examples of recognised and unrecognised (crossed) faces.

chical linking from coarse to fine scales, as already applied in the segregation process, has not been applied. Such an extension can lead to better recognition rates, especially when multiple views (frontal, $3 / 4$ and side) of all persons are included as templates in "memory." In addition, the multi-scale keypoint representation [15], which has not been employed here, will contribute very important information.

All multi-scale processing and the representations, including keypoints, are restricted to area V1. On the other hand, the Deco and Rolls scheme [4], with ventral and dorsal data streams, necessary for obtaining translation and scale invariance through projections via areas V2, V4 etc., is solely based on responses of simple cells. In the future, this scheme must be based on features extracted in V1, and further multi-scale processing can be added in higher areas V2 and V4. We expect that such extensions in adaptive up and down projections will lead to much better results. Afterall, our visual system does not have any difficulty in telling persons apart!

Acknowledgements: This investigation is partly financed by PRODEP III Medida 5, Action 5.3, and by the FCT program POSI, framework QCA III. The Stirling images are available at http://pics.psych.stir.ac.uk/ 


\section{References}

1. Wässle, H.: Parallel processing in the mammalian retina. Nature Rev. Neuroscience 10 (2004) 747-757

2. Berson, D.: Strange vision: ganglion cells as circadian photoreceptors. TRENDS in Neurosciences 26 (2003) 314-320

3. Kastner, S.: Beyond a relay nucleus: new views on the human LGN. Perception Suppl. 34 (2005) 36

4. Deco, G., Rolls, E.: A neurodynamical cortical model of visual attention and invariant object recognition. Vision Res. (2004) 621-642

5. Rensink, R.: The dynamic representation of scenes. Visual Cogn. 7 (2000) 17-42

6. Rasche, C.: The making of a neuromorphic visual system. Springer (2005)

7. Hubel, D.: Eye, brain and vision. Scientific American Library (1995)

8. Heitger et al., F.: Simulation of neural contour mechanisms: from simple to end-stopped cells. Vision Res. 32 (1992) 963-981

9. Grigorescu, C., Petkov, N., Westenberg, M.: Contour detection based on nonclassical receptive field inhibition. IEEE Tr. IP 12 (2003) 729-739

10. Rodrigues, J., du Buf, J.: Visual cortex frontend: integrating lines, edges, keypoints and disparity. Proc. Int. Conf. Image Anal. Recogn. Springer LNCS 3211 (2004) 664-671

11. Elder, J., Zucker, S.: Local scale control for edge detection and blur estimation. IEEE Tr. PAMI 20 (1998) 699-716

12. van Deemter, J., du Buf, J.: Simultaneous detection of lines and edges using compound Gabor filters. Int. J. Patt. Recogn. Artif. Intell. 14 (1996) 757-777

13. Fleet, D., Jepson, A., Jenkin, M.: Phase-based disparity measurement. CVGIP: Image Understanding 53 (1991) 198-210

14. Zhaoping, L.: V1 mechanisms and some figure-ground and border effects. J. of Physiology (2003) 503-515

15. Rodrigues, J., du Buf, J.: Multi-scale keypoints in V1 and face detection. 1st Int. Symp. Brain, Vision and Artif. Intell., Naples (Italy) Springer LNCS 3704 (2005) 205-214

16. Yang, M., Kriegman, D., Ahuja, N.: Detecting faces in images: A survey. IEEE Tr. PAMI 24 (2002) 34-58

17. Petkov, N., Kruizinga, P., Lourens, T.: Biologically motivated approach to face recognition. Proceeding of International Workshop on Artificial Neural Networks' (1993) 68-77

18. du Buf, J.: Responses of simple cells: events, interferences, and ambiguities. Biol. Cybern. 68 (1993) 321-333

19. Krüger, N., Peters, G.: Object recognition with banana wavelets. Proc. 5th Europ. Symp. Artificial Neural Networks (1997) 61-66

20. Heath, M., Sarkar, S., Sanocki, T., Bowyer, K.: A robust visual method for assessing the relative performance of edge-detection algorithms. IEEE Tr. PAMI 19 (2000) 1338-1359

21. Lindeberg, T.: Scale-space theory in computer vision. Kluwer Academic Publishers, Dordrecht, The Netherlands (1994)

22. du Buf, J.: Ramp edges, Mach bands, and the functional significance of simple cell assembly. Biol. Cybern. 70 (1994) 449-461

23. du Buf, J., Fischer, S.: Modeling brightness perception and syntactical image coding. Optical Eng. 34 (1995) 1900-1911

24. Bar, M.: A cortical mechanism for triggering top-down facilitation in visual object recognition. J. Cogn. Neuroscience (2003) 600-609 